\section{Consensos sobre o papel do gestor estadual na regionalização da assistência à saúde no Sistema Único de Saúde (SUS)}

\section{Consensus regarding the role of the State manager in the regionalization of health care in Brazilian National Health System (SUS)}

Yluska Almeida Coelho dos Reis 1

Eduarda Ângela Pessoa Cesse 2

Eduardo Freese de Carvalho 3

1-3 Laboratório de Apoio à Municipalização. Centro de Pesquisas Aggeu Magalhães. Fundação Oswaldo Cruz. Av. Prof. Morais do Rego. Cidade Universitária, s.n. $4^{\circ}$ andar. Recife, PE, Brasil. CEP: 50.670-420. Caixa Postal: 7472. E-mail: yluska@cpqam.fiocruz.br

\begin{abstract}
Objectives: to identify points of agreement regarding the role of the State manager in the regionalization of health care in Brazilian National Health System (SUS).

Methods: a theoretical framework and evaluation grid were drawn up to evaluate the role of the State manager in regionalization. These were ratified by specialists, who confirmed the importance of the indicators chosen to establish the existence of consensus or the lack of it, on the basis of previously established cut-off points, using the Consensus Conference technique.

Results: the evaluation grid resulting from the consensus achieved involves three levels of analysis (government, management and care), which are broken down into six categories comprising 14 criteria and 82 indicators exploring the responsibility of the State manager of the SUS vis-à-vis the regionalization of health care. There was not a consensus regarding only four of these indicators, suggesting that the grid adopted is an efficient instrument for the evaluation of the regionalization of health care in SUS care and covers the various levels of this healt care intervention.

Conclusions: the consensus now established, however provisional, may be taken as an extremely useful point of reference for further research and could serve as the basis for future evaluations of the implementation of regionalization, in such a way as to allow for comparative studies to be carried out.
\end{abstract}

Key words Health evaluation, Decentralization, Regional health planning, Health management, Delivery of health care, Single health system

\section{Resumo}

Objetivos: identificar os consensos acerca do papel do gestor estadual na regionalização da assistência à saúde no SUS.

Métodos: foram elaborados um modelo teóricológico e uma matriz de avaliação acerca das atribuições do gestor estadual na condução da regionalização. Essa matriz foi submetida à apreciação de especialistas que julgaram a importância dos indicadores de análise definidos, estabelecendo-se o consenso ou o dissenso a partir de pontos de corte previamente definidos, utilizando-se como técnica a Conferência de Consenso.

Resultados: a matriz de avaliação resultante do consenso apresenta três níveis de análise (governo, gestão e assistência), que estão desmembrados em seis dimensões compostas de 14 critérios e 82 indicadores de avaliação que exploram as responsabilidades do gestor estadual do SUS frente à regionalização da saúde. Do total de indicadores, apenas quatro não apresentaram consenso, o que indica que a matriz se mostra um instrumento eficiente para avaliação da regionalização da assistência no SUS, abordando as diferentes dimensões dessa intervenção em saúde.

Conclusões: apesar de provisório, o consenso ora estabelecido pode constituir um referencial de elevada utilidade para a realização de pesquisas, podendo servir de base para a realização de investigações avaliativas sobre a implantação dos processos de regionalização, de forma a permitir maior comparabilidade entre os estudos.

Palavras-chave Avaliação em Saúde, Descentralização, Regionalização, Gestão em saúde, Assistência à Saúde, Sistema Único de Saúde 


\section{Introdução}

A estratégia de descentralização da saúde adotada na primeira década de implantação do Sistema Único de Saúde (SUS) proporcionou inúmeros avanços com a incorporação, pelos municípios, da responsabilidade pela provisão de serviços em seus territórios. Todavia, permanecem, na atualidade, problemas relativos à iniquidade na oferta e no acesso, à intensa fragmentação e à desorganização dos serviços do SUS. Isso se deve, em grande parte, ao fato de que muitos dos problemas em saúde extrapolam os limites administrativos e a governabilidade das instâncias municipais, requerendo a consolidação de sistemas regionais de saúde.1-3

Apesar de incorporados conjuntamente às diretrizes operacionais do SUS, os conceitos de descentralização e regionalização não operaram, necessariamente, em sentidos convergentes ao longo do tempo. Tratadas de forma antagônica ou tomando-se a regionalização como um instrumento da descentralização, a tensão entre ambas as diretrizes foi acentuada. ${ }^{2}$ De um lado, o processo de descentralização enfocou os milhares de municípios existentes, o que na definição de Lucchese 4 provocou a atomização de sistemas municipais de saúde, ou a municipalização autárquica, na concepção de Mendes, ${ }^{1}$ conduzindo a um modelo de assistência municipalista, no qual a atenção médica é expandida sem nenhuma articulação regional. De outro, a regionalização, mais tardiamente, veio agregar maior responsabilidade das instâncias estaduais, cujo papel esteve negligenciado ou insuficientemente definido pelo arcabouço do SUS até o início da década de 2000. É nesse momento que a regionalização ganha força no debate setorial, institucionalizando-se como política capaz de reverter o municipalismo radical em curso. ${ }^{3}$

Contudo, conforme destacam Hartz e Contandriopoulos, 5 o modelo de um "sistema sem muros", regionalizado, integral e resolutivo, capaz de eliminar as barreiras de acesso entre os níveis de atenção, ainda que consensual em seus princípios, tem se mostrado de difícil operacionalização e demanda por estudos de avaliação que possam contribuir para sua efetiva implantação.

Nesse contexto, coloca-se como etapa indispensável à avaliação desse sistema, a descrição das intervenções em saúde, que de acordo com Contandriopoulos et al. 6 constituem "o conjunto dos meios (físicos, humanos, financeiros, simbólicos) organizados em um contexto específico, em um dado momento, para produzir bens ou serviços com o objetivo de modificar uma situação problemática".
Para Chen, ${ }^{7}$ uma intervenção em saúde é constituída de aspectos estruturais e operacionais, mas também de uma teoria subjacente, carregada de valor, que incorpora saberes científicos e também saberes práticos dos grupos implicados na avaliação, que se fazem importantes conhecer.

Contudo, cabe destacar a pouca clareza com que os documentos oficiais apresentam a teoria das intervenções em saúde, bem como a falta de consenso entre os atores envolvidos sobre sua operacionalização e resultados esperados, ${ }^{8}$ o que gera dificuldades aos processos de implantação e consequentemente à avaliação destes. Nesse sentido, o desenho de um modelo teórico-lógico permite explicitar a racionalidade subjacente da intervenção a ser avaliada, o problema, a população-alvo e os atributos necessários e suficientes para produzirem isolada ou integradamente os efeitos esperados. 9

No tocante à regionalização da assistência à saúde, espera-se que a sistematização de um modelo teórico-lógico possa servir de referência para estudos avaliativos acerca da implantação dos processos de regionalização em curso no Brasil, permitindo aos pesquisadores trabalhar com diversas estratégias metodológicas no sentido de aferir em que medida esse modelo está sendo alcançado, e ampliando as chances de comparabilidade entre os estudos.

Partindo desse pressuposto, este estudo teve como objetivo identificar os consensos acerca do papel do gestor estadual na condução da política de regionalização do SUS, tomando como referência seu modelo teórico-lógico. Constitui a primeira etapa do estudo de análise de implantação da regionalização da assistência à saúde em desenvolvimento no Estado de Pernambuco.

\section{Métodos}

Elaborou-se o modelo teórico-lógico da regionalização da assistência à saúde a partir da revisão da literatura especializada2,5,10-19 e do referencial normativo do SUS (Tabela 1). O modelo teóricológico, representado na Figura 1, demonstra de forma esquemática a teoria subjacente à regionalização, seus mecanismos operacionais e sua relação com o contexto e com os resultados a que se propõe. A partir deste modelo foi sistematizada uma matriz de avaliação para análise do papel do gestor estadual na regionalização da assistência à saúde. Esta matriz foi estruturada em três níveis de análise:governo, gestão e assistência. 
Referencial normativo do Sistema Único de Saúde utilizado na sistematização do modelo lógico da regionalização da assistência à saúde no SUS.

Leis

Decreto

Portarias

Resoluções

Manuais técnicos Caderno do programa nacional de avaliação de serviços de saúde. Edição 2004/2005. Diretrizes para a implantação de complexos reguladores. Normas e manuais técnicos. Ministério da Saúde. 1 ed.; 2006.

Propostas

Lei $N^{\circ} 8.080$, de 19 de setembro de 1990. Dispõe sobre as condições para a promoção, proteção e recuperação da saúde, a organização e o funcionamento dos serviços correspondentes e dá outras providências.

Lei 8.142. Dispõe sobre a participação da comunidade na gestão do Sistema Único de Saúde e sobre as transferências intergovernamentais de recursos financeiros na área da saúde e dá outras providências.

Decreto n. 1.651, de 28 de setembro de 1995. Regulamenta o sistema nacional de auditoria no âmbito do Sistema Único de Saúde.

Portaria GM/MS, n. 2.203, de 06 de novembro de 1996. Norma Operacional Básica do SUS - NOB 96. Redefine o modelo de gestão para o processo de consolidação do SUS e o efetivo cumprimento dos princípios e diretrizes que o orientam.

Portaria GM/MS, n. 95, de 26 de janeiro de 2001. Norma Operacional de Assistência à Saúde - NOAS-SUS 01/2001. Amplia as responsabilidades dos municípios na Atenção Básica; define o processo de regionalização da assistência; cria mecanismos para o fortalecimento da capacidade de gestão do Sistema Único de Saúde e procede à atualização dos critérios de habilitação de estados e municípios.

Portaria GM/MS, n. 373, de 27 de fevereiro de 2002. Norma Operacional de Assistência à Saúde - NOASSUS 01/2002. Amplia as responsabilidades dos municípios na atenção básica; estabelece o processo de regionalização como estratégia de hierarquização dos serviços de saúde e de busca de maior equidade; cria mecanismos para o fortalecimento da capacidade de gestão do Sistema Único de Saúde e procede à atualização dos critérios de habilitação de estados e municípios.

Portaria GM/MS n. 399, de 22 de fevereiro de 2006. Divulga o pacto pela saúde 2006 - Consolidação do SUS.

Portaria MS/GM n. 699, de 30 de março de 2006. Regulamenta as diretrizes operacionais dos pactos pela vida e de gestão.

Portaria GM/MS n. 598, de 23 de março de 2006. Define que os processos administrativos relativos à gestão do SUS sejam definidos e pactuados no âmbito das Comissões Intergestores Bipartite.

Portaria GM/MS n. 204, de 29 de janeiro de 2007. Regulamenta o financiamento e a transferência dos recursos federais para as ações e os serviços de saúde, na forma de blocos de financiamento, com o respectivo monitoramento e controle.

Portaria GM/MS n. 1.101, de 12 de junho de 2002. Estabelece os parâmetros de cobertura assistencial no âmbito do Sistema Único de Saúde (SUS).

Portaria GM/MS n. 2.923, de 09 de junho de 1998. Institui o programa de apoio à implantação dos sistemas estaduais de referência hospitalar em atendimento de urgência e emergência.

Portaria GM/MS n. 479, de 14 de abril de 1999. Altera os mecanismos para a implantação dos sistemas estaduais de referência hospitalar em atendimento de urgência e emergência.

Portaria MS n. 2.048 de 05 de novembro de 2002. Estabelece os princípios e diretrizes dos sistemas estaduais de urgência e emergência, as normas e critérios de funcionamento, classificação e cadastramento de serviços, entre outros.

Portaria MS/GM n. 635, de 10 de novembro de 2005. Publica o Regulamento técnico para a implantação e a operacionalização do programa de reestruturação dos hospitais filantrópicos no SUS.

Portaria MS/GM n. 1.721, de 21 de setembro de 2005. Cria o programa de reestruturação e contratualização dos hospitais filantrópicos no SUS.

Portaria MS/GM n. 1.996, de 20 de agosto de 2007. Dispõe sobre as diretrizes para a implementação da política nacional de educação permanente em saúde.

Portaria MS/GM n. 2.048, de 03 de setembro de 2009. Aprova o regulamento do Sistema Único de Saúde.

Resolução RDC/Anvisa n. 50, de 21 de fevereiro 2002. Regulamento para planejamento, programação elaboração e avaliação de projetos físicos de estabelecimentos de assistência à saúde.

Resolução RDC/Anvisa n. 189, de 18 de julho de 2003. Dispõe sobre a regulamentação dos procedimentos de análise, avaliação e aprovação dos projetos físicos de estabelecimentos de saúde no sistema nacional de vigilância sanitária, altera o regulamento técnico aprovado pela RDC $n^{\circ} 50$, de 21 de fevereiro de 2002 e dá outras providências.

Política Nacional de Informação e Informática em Saúde (PNIIS). Proposta; 2004.

SUS= Sistema Único de Saúde 


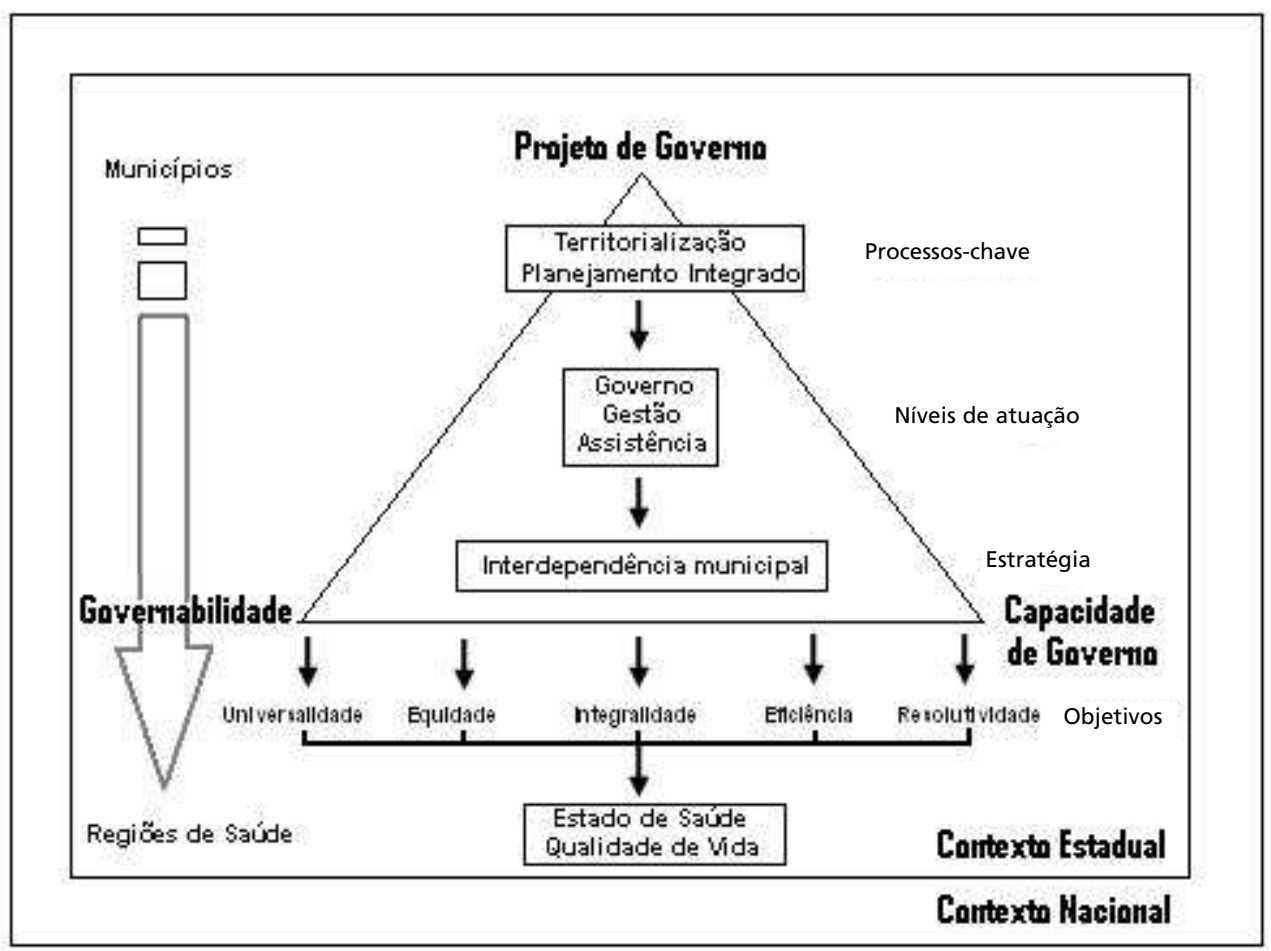

O nível de análise governo é composto por duas dimensões e seus critérios de avaliação: projeto estadual de regionalização (territorialização da saúde; gestão compartilhada/co-gestão; fortalecimento da capacidade institucional) e financiamento (promoção da equidade; alocação de recursos). O nível gestão é constituído pelas seguintes dimensões e critérios: acesso e continuidade do sistema de saúde (redes integrais de atenção à saúde; política de recursos humanos; planejamento, monitoramento e avaliação; regulação assistencial; tecnologias de gestão) e participação e controle social (participação da sociedade na definição e acompanhamento da política regional de saúde). E o nível assistência pelas dimensões e critérios: qualidade da assistência (infraestrutura dos serviços; qualidade técnica) e educação em saúde (política de educação permanente).

Cada um dos critérios de avaliação da matriz agrupa um conjunto de indicadores de avaliação que buscam explicitar as atribuições inerentes ao gestor estadual na condução do processo de regionalização da assistência à saúde.

A matriz de avaliação foi submetida a um consenso entre especialistas, através da técnica denominada de conferência de consenso, 16 escolhida por conciliar tanto a discussão entre especialistas convidados quanto a preservação do anonimato, ocorrida entre agosto e outubro de 2009. Foram convidados 15 especialistas: gestores e técnicos municipais, estaduais e do nível federal (8), pesquisadores (4) e especialistas em avaliação (3). Estes foram selecionados a partir da sua inserção nas áreas de atuação em gestão e planejamento, regulação da assistência, descentralização e regionalização em saúde e pela produção científica sobre o tema em questão.

A conferência de consenso foi organizada em três etapas. Na primeira, a matriz de avaliação foi encaminhada via correio eletrônico e cada especialista procedeu a uma análise individual das dimensões, critérios e indicadores de avaliação propostos, atribuindo, numa escala de 0 a 10 , a importância de cada indicador para a avaliação da gestão estadual no tocante à regionalização. Todos os especialistas convidados encaminharam sua análise da matriz.

$\mathrm{Na}$ etapa seguinte foi realizada uma oficina com os especialistas, a qual possibilitou a discussão acerca das dimensões, critérios e indicadores definidos, com o objetivo de promover a troca de 
experiências e o confronto de opiniões, principalmente em torno dos pontos de maior divergência apresentados como resultado da etapa anterior. A discussão foi direcionada no sentido de avaliar a pertinência e a suficiência dos critérios e indicadores de avaliação como atribuições da esfera estadual de gestão. Nesta etapa, compareceram nove especialistas, dos quais oito haviam participado do primeiro momento.

A terceira e última etapa consistiu em uma nova análise individual da matriz reformulada pelo debate, nos moldes da primeira etapa. Dos nove especialistas, oito encaminharam a matriz reavaliada.

Para a identificação do consenso foram calculados a média e o desvio padrão para cada indicador, critério e dimensão de avaliação da matriz, a partir da pontuação conferida pelos especialistas. A média proporcionou mensurar o grau de importância de cada componente da matriz, enquanto o desviopadrão permitiu estimar o grau de consenso. Com base na classificação proposta por Souza et al., $16 \mathrm{o}$ ponto de corte para a média foi fixado em 7 e o ponto de corte para o desvio-padrão fixado em 3 . Com base nisso, os componentes da matriz foram categorizados em quatro grupos: consensualmente importantes; consensualmente pouco importantes; importantes com dissenso e pouco importantes com dissenso (Tabela 2).

Os componentes importantes e consensuais foram aqueles entendidos como essenciais no âmbito de atribuições da gestão estadual no que concerne à regionalização da assistência à saúde e que, por isso, devem integrar sua matriz de avaliação.

Este estudo foi aprovado pelo Comitê de Ética em Pesquisa do Centro de Pesquisas Aggeu Magalhães em 2008.

Tabela 2

Classificação quanto ao consenso.

\begin{tabular}{lcc}
\hline Média & Desvio-padrão & Classificação do critério \\
\hline & & \\
Igual ou maior que 7 & Menor que 3 & Consensualmente importante \\
Menor que 7 & Menor que 3 & Consensualmente pouco importante \\
Igual ou maior que 7 & Igual ou maior que 3 & Importante com dissenso \\
Menor que 7 & Igual ou maior que 3 & Pouco importante com dissenso \\
\hline
\end{tabular}

Fonte: Souza et al. 16

\section{Resultados}

Observou-se que em todas as etapas da conferência prevaleceu o consenso acerca de se avaliar as atribuições do gestor estadual na regionalização do ponto de vista dos três níveis de análise identificados. Em geral, as dimensões e critérios também se mostraram consensuais, embora tenha se identificado a partir da discussão a necessidade de torná-los mais objetivos. Em relação aos indicadores, as principais mudanças ocorridas se deram em termos qualitativos, de forma a torná-los mais precisos como ferramenta para avaliação.

A primeira versão da matriz de avaliação encaminhada aos especialistas era composta por 3 níveis de análise, 9 dimensões, 19 critérios e 83 indicadores. Após a revisão, a matriz, ainda com os mesmos três níveis de análise, passou a apresentar 6 dimensões, 14 critérios e 82 indicadores de avaliação. A permanência de uma grande quantidade de indicadores reflete a complexidade do fenômeno sob estudo. Além disso, as dimensões, critérios e indicadores que compõem a matriz de avaliação apresentam zonas de sobreposição, na medida em que são complementares e interdependentes.

Como resultado da primeira etapa da Conferência obteve-se oito indicadores classificados como não consensuais e/ou não importantes entre os 83 indicadores que integraram a matriz nesse momento. Além disso, destaca-se que destes, dezoito indicadores, mesmo classificados como consensuais e importantes, obtiveram desvio padrão entre 2,0 e 2,9 , ou seja, mais próximos do limite de serem considerados não consensuais.

O resultado do consenso na etapa final (Tabela 3 ), apresentou quatro indicadores não consensuais e/ou não importantes dos 82 que integram a matriz, todos pertencentes ao nível de análise gestão, o que demonstra a importância relativa da segunda etapa da conferência (a discussão entre os especialistas) 


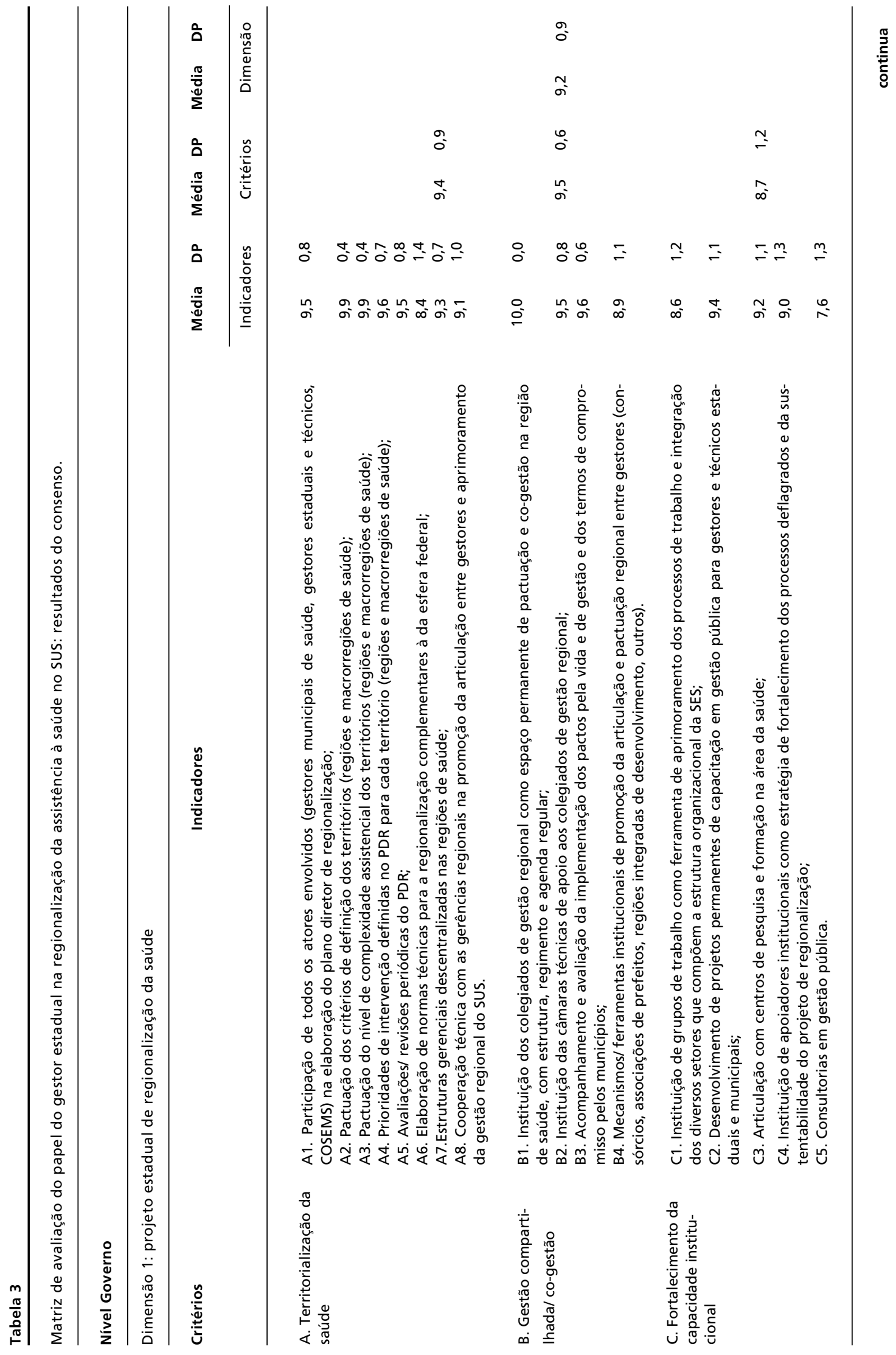

S162 Rev. Bras. Saúde Matern. Infant., Recife, 10 (Supl. 1): S157-S172 nov., 2010 


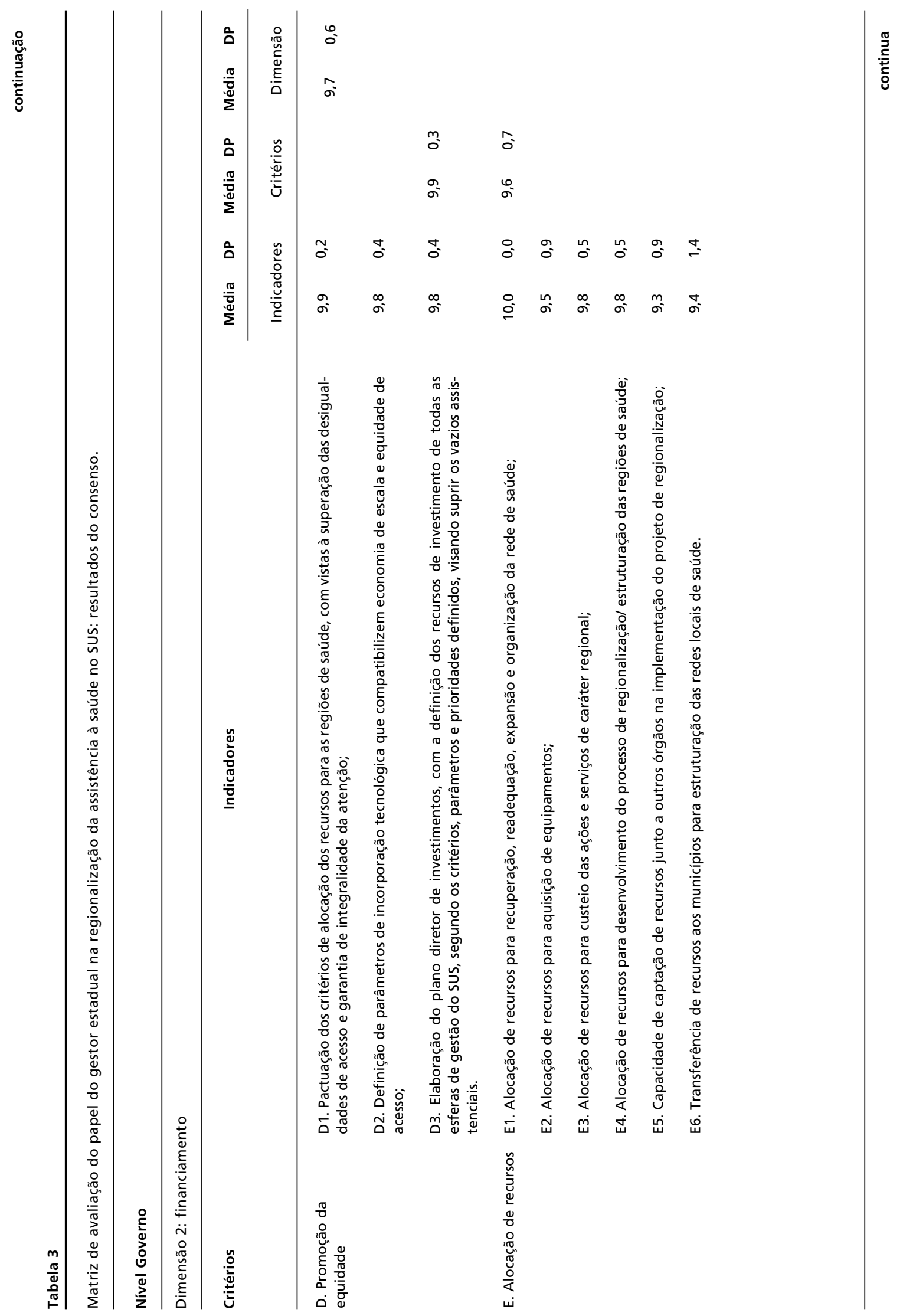




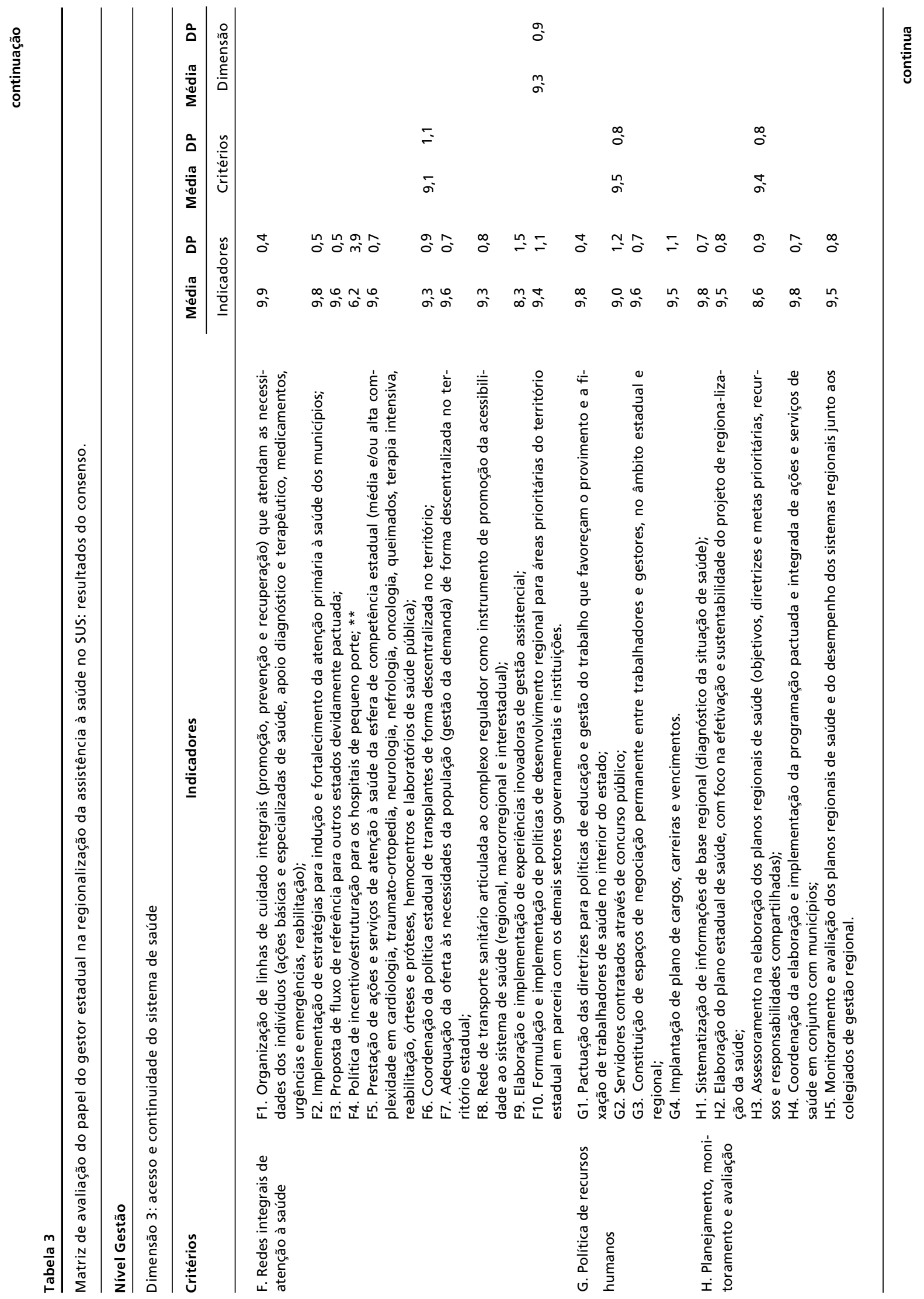




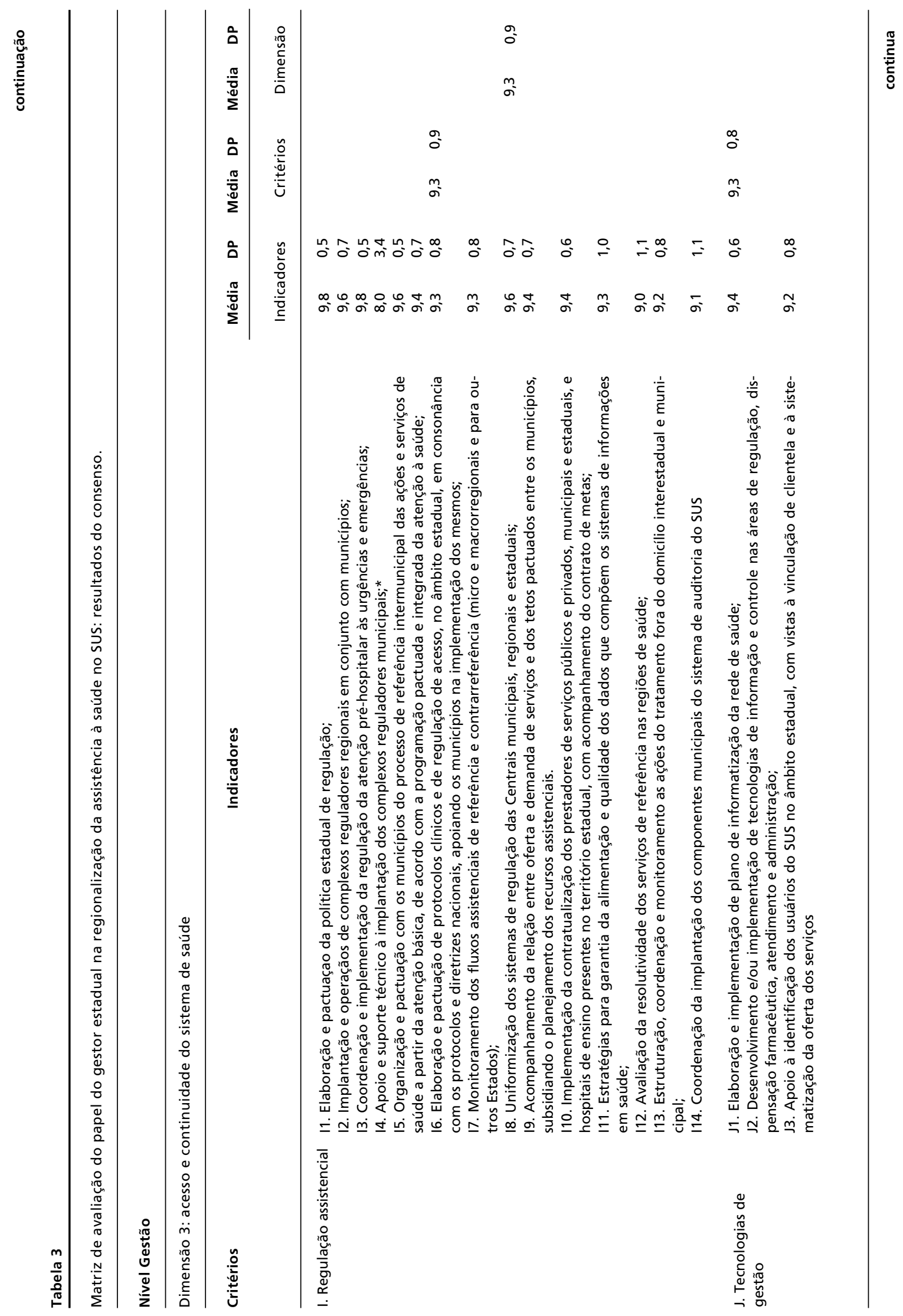




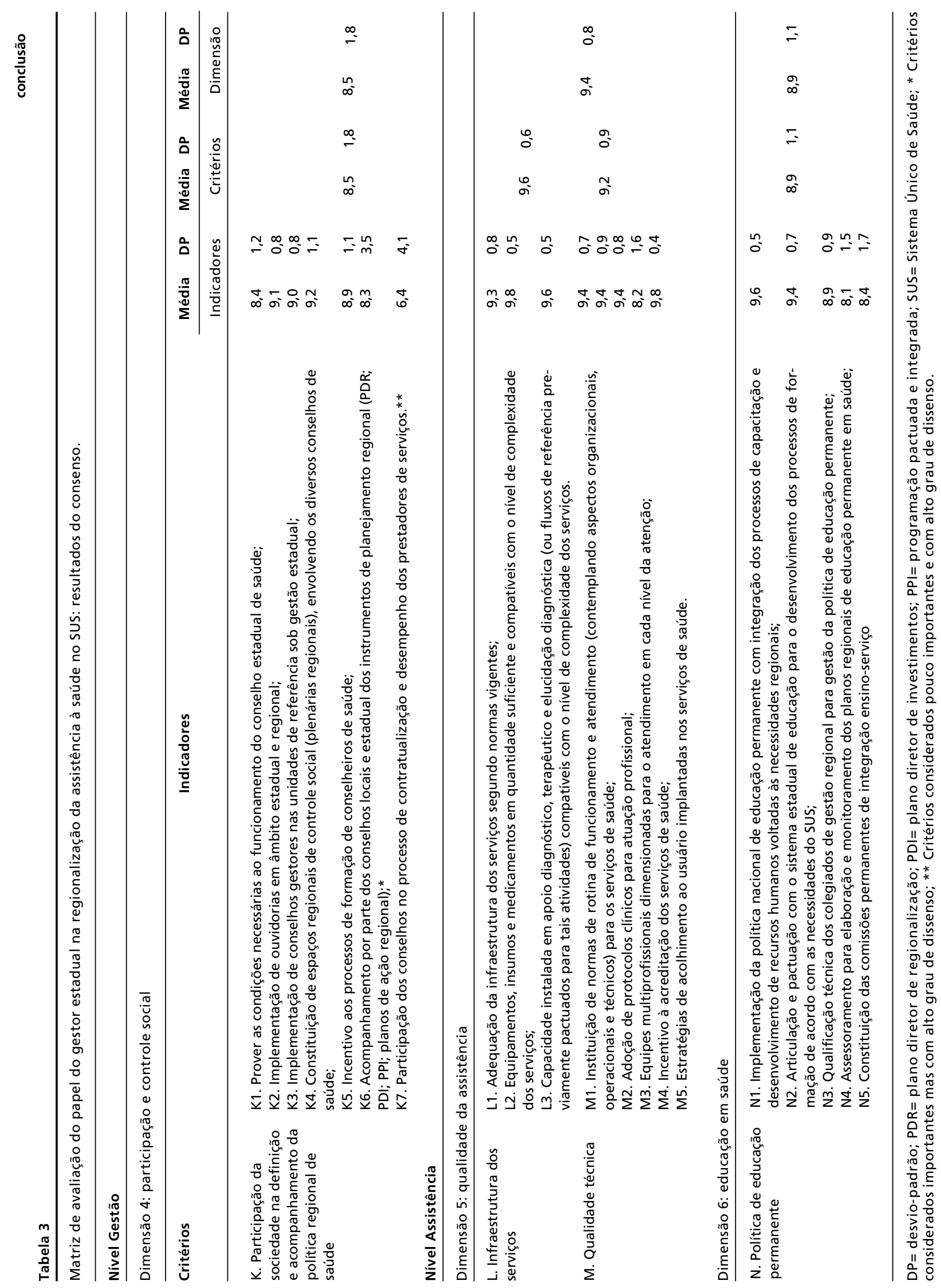


sobre o estabelecimento do consenso. Foram considerados não consensuais e pouco importantes: a política de investimentos para hospitais de pequeno porte (média $=6,2$ e desvio-padrão $=3,9$ ) e a participação dos Conselhos na contratualização de prestadores de serviços (média=6,4 e desvio-padrão=4,1). E como não consensuais, ainda que importantes: o suporte à implantação de complexos reguladores municipais (média $=8,0$ e desvio-padrão $=3,4$ ) e o acompanhamento dos instrumentos de planejamento regional pelos Conselhos (média $=8,3$ e desviopadrão=3,5).

Entre os indicadores importantes e consensuais, destacam-se a instituição de colegiados de gestão regional (média $=10,0$ e desvio-padrão $=0,0$ ) e a alocação de recursos para recuperação, readequação, expansão e organização da rede de saúde (média $=10,0$ e desvio-padrão $=0,0$ ), ou seja, com grau máximo de consenso entre os especialistas.

Em relação às dimensões de análise da matriz (Tabela 3), a que obteve a maior importância relativa foi a dimensão financiamento (média $=9,7$ e desviopadrão $=0,6$ ), seguida pela dimensão qualidade da assistência (média $=9,4$ e desvio-padrão $=0,8$ ) e acesso e continuidade do sistema de saúde (média $=9,3$ e desvio-padrão=0,9). Destacam-se com as menores importâncias relativas e maiores graus de divergência as dimensões participação e controle social (média $=8,5$ e desvio-padrão $=1,8$ ) e educação em saúde (média=8,9 e desvio-padrão=1,1) em relação às demais dimensões.

\section{Discussão}

\section{Nível de análise: Governo}

Nesse nível de análise, todos os critérios e indicadores de avaliação que compõem a dimensão projeto estadual de regionalização e a dimensão financiamento foram considerados importantes e consensuais no julgamento do grupo de especialistas. Um dos aspectos centrais da discussão considerou que a regionalização precisa ser assumida como projeto de governo, não se limitando apenas a uma divisão espacial do território estadual sem relação entre as partes. Entendeu-se que mais importante do que verificar a existência do plano diretor de regionalização e demais instrumentos de planejamento regional, é avaliar o nível de participação dos atores e a consistência desses instrumentos no que diz respeito à pactuação dos critérios de definição das regiões de saúde, que obteve o maior grau de importância entre os indicadores desse nível de análise.
Para os especialistas, a capacidade de articulação do gestor estadual em promover o envolvimento integral desses atores e em provocar o sentimento de pertencimento regional é uma tarefa complexa, mas sem dúvida um fator crucial para o desenvolvimento de uma regionalização cooperativa, pois conforme referem Pestana e Mendes ${ }^{14}$ é no espaço regional de pertencimento econômico, social, cultural e sanitário, que se constroem redes de relações intermunicipais e se recupera uma escala necessária ao desenvolvimento econômico e social.

No tocante à gestão compartilhada, foi consenso entre os especialistas que a instituição dos colegiados de gestão regional, das câmaras técnicas e dos termos de compromisso, como ferramentas de gestão, têm o potencial de promover a regionalização cooperativa definidas no pacto de gestão do SUS. Contudo, também foi consenso que o gestor estadual possa estimular a adoção de diferentes ferramentas na promoção de uma articulação regional, como os consórcios, associações de prefeitos, regiões integradas de desenvolvimento, entre outros, pois a organização da assistência via regionalização, conforme destacam Artmann e Rivera, 20 é um processo que exige flexibilidade, não sendo passível de se resolver pela aplicação de um instrumento normativo homogêneo. Em um país com grandes dimensões e diferenças regionais, é necessário pensar com criatividade e contextualizar os parâmetros através de adaptações viáveis, que resultem em impactos positivos sobre a situação problemática.

Sobre os aspectos normativos, cabe destacar também que a elaboração de normas técnicas complementares a da gestão federal por parte do gestor estadual foi um aspecto inicialmente divergente. De um lado, havia o ponto de vista de que a normatização excessiva no SUS é prejudicial ao funcionamento do sistema de saúde e, de outro, que sendo a regionalização uma função compartilhada entre os níveis de gestão, as normas complementares poderiam contribuir abordando as especificidades locais, ponto de vista que acabou prevalecendo na etapa final.

No que se refere ao fortalecimento da capacidade institucional, o debate esteve concentrado sobre o fato de que as relações de qualificação da gestão acabam se dando de forma muito externa. Sem desprezar a necessidade de articulação com centros de formação para o desenvolvimento de projetos permanentes de capacitação da gestão pública, os especialistas também consideram relevante a adoção de mecanismos internos de fortalecimento da capacidade institucional, como apoiadores 21 que atuem 
transversalmente e intersetorialmente, a fim de conferir maior sustentabilidade ao projeto de regionalização.

Ainda no tocante à capacidade institucional, as falas de alguns especialistas apontaram para a dificuldade de integração das consultorias com o projeto institucional da saúde. Para eles, muitas vezes, a opção do gestor por uma consultoria acaba desconsiderando o acúmulo da equipe técnica que gerencia o processo, provocando conflitos e desestímulo quanto à sua condução. Já para outros, ainda que as experiências vivenciadas tenham sido mal sucedidas, as consultorias são instrumentos necessários e capazes de provocar efeitos positivos quando bem direcionadas, entendimento este que parece ter contribuído para tornar o indicador consensual na última análise.

$\mathrm{Na}$ dimensão financiamento, um aspecto que se faz relevante destacar refere-se à forma de alocação dos recursos financeiros na saúde. Sabe-se dos grandes desafios ainda existentes em relação ao financiamento do setor saúde, que além de insuficiente, ainda se apresenta bastante atrelado à oferta $\mathrm{e}$ produção de serviços. 22 De acordo com os participantes da conferência não se pode afirmar que os recursos estão alocados onde mais se precisa. Mas, é certo que recebe mais quem possui mais serviços de saúde. É nesse sentido que os especialistas consideram de grande relevância a atuação do gestor estadual frente à pactuação de critérios de alocação de recursos, que contribuam de forma mais efetiva para a superação das desigualdades entre as regiões de saúde e para a garantia da equidade e da integralidade da atenção.

Para os especialistas consultados é imprescindível que o gestor estadual contribua para a consolidação de um financiamento solidário entre as três esferas de gestão, equacionando os recursos do ponto de vista da reestruturação dos serviços de saúde e da organização de sistemas regionais de saúde, a partir da sua capacidade de captar recursos junto à esfera federal e de alocar os recursos com base nos critérios e parâmetros definidos.

\section{Nível de análise: Gestão}

Esse foi o único nível de análise que apresentou pontos de dissenso entre os especialistas. $\mathrm{Na}$ dimensão acesso e continuidade do sistema de saúde, um aspecto relevante da discussão entre os especialistas aponta para o fortalecimento do papel do gestor estadual na organização de redes integrais de atenção à saúde, desde a sua atuação como fomentador da atenção primária, passando pelas estratégias de organização de linhas de cuidados integrais, até a prestação de serviços de maior complexidade.

Nessa perspectiva, foi consenso que a organização de linhas de cuidado consiste em uma ferramenta potente de aproximação da integralidade, desenhando o caminho a ser percorrido pelos usuários na sua busca por cuidado integral, de forma horizontal ou em círculo, e não mais com base na pirâmide, como é tradicionalmente representado o sistema de saúde, com definição razoavelmente clara das missões e das responsabilidades de cada unidade integrante da rede. 23,24

$\mathrm{Na}$ visão dos especialistas, no tocante à regionalização, também se faz relevante avaliar o papel do gestor estadual na implementação e organização da rede local, especialmente pela adoção de estratégias de indução e fortalecimento da atenção primária que contribuam para o aumento da resolutividade local da assistência, bem como pela pactuação dos fluxos assistenciais de referência para outros Estados.

Ainda no tocante à organização de redes integrais de atenção à saúde, cabe destacar que a política de incentivo/estruturação para hospitais de pequeno porte foi considerada pouco importante e com elevado grau de discordância entre os especialistas. Isso porque, na visão de alguns, pode conduzir a distorções de operacionalização por parte dos gestores, disseminando serviços hospitalares que agregam pouca resolutividade e são de alto custo. Para outros, esses hospitais podem ser elementos estratégicos, considerando sua elevada participação no sistema de saúde brasileiro (mais de 4000 estabelecimentos) e a possibilidade de reorientação do seu papel ao lado da Estratégia Saúde da Família (ESF), sendo objeto de política específica, editada em 2004 pelo Ministério da Saúde. 25

Um indicador de avaliação também problematizado pelos especialistas e que implica maiores oportunidades de acesso aos serviços constitui a rede de transporte sanitário. Na primeira análise da matriz esse indicador não obteve consenso entre os participantes, talvez pela carência de uma concepção ordenada de sistema de transporte sanitário no SUS, o que fez com que fosse associada a práticas clientelistas como a "ambulancioterapia" utilizada por inúmeros gestores. O indicador passou a ser relevante e consensual após a discussão entre os especialistas, quando se tornou claro seu papel na organização de redes regionais e na garantia do acesso, atendida a necessidade de um controle articulado ao complexo regulador das regiões de saúde. 14

É importante ressaltar que, para os especialistas consultados, todos esses aspectos devem estar inseridos num contexto de forte desenvolvimento 
regional expressa na política de governo, dentro de uma visão de conjunto e buscando atender às exigências peculiares a cada região. Isso depende em grande medida da capacidade do gestor estadual em implementar políticas públicas que, em adequação às prioridades regionais, ${ }^{2}$ estimulem o desenvolvimento econômico e social. Só assim será possível impactar sobre as desigualdades inter-regionais e por consequência sobre as desigualdades em saúde, pretendidas pelo modelo de regionalização.

Quanto às atribuições do gestor estadual na política de recursos humanos, entendeu-se que esta deve ser implementada no plano estadual de forma a garantir a fixação de profissionais especializados e também generalistas no interior do Estado. Para o grupo de especialistas, o gestor estadual precisa trabalhar uma política de recursos humanos contextualizada regionalmente, dialogando com os municípios no estabelecimento de diretrizes gerais que produzam condições de maior igualdade entre as regiões na fixação de profissionais de saúde.

Fica evidente também o consenso de que os compromissos assumidos entre os gestores precisam ser formalizados por meio de instrumentos de pactuação e de planejamento, explicitando as responsabilidades dos gestores e o conjunto de objetivos e ações que contribuirão para a garantia do acesso e da integralidade da atenção. Esse sistema de planejamento regional inclui o monitoramento e avaliação das ações implementadas e dos resultados alcançados, como instrumento estratégico de gestão do SUS e de fortalecimento do próprio planejamento, na medida em que lhe confere direcionalidade e precisão. 26

Ainda na dimensão acesso e continuidade do sistema de saúde, foi consenso entre os especialistas, a importância de se avaliar o papel do gestor estadual na definição de uma política estadual de regulação, que estabeleça as diretrizes para a implantação e operacionalização de complexos reguladores regionais, para a pactuação e o monitoramento dos fluxos assistenciais, para a contratualização dos prestadores de serviços, para a uniformização dos sistemas de regulação em todo o território estadual, para a construção de protocolos clínicos e de regulação do acesso e para a implantação dos componentes municipais de auditoria, aspectos esses que proporcionam a instituição de uma linguagem comum em termos de regulação assistencial.

Era de se esperar, dada a estreita relação com os demais, que o indicador apoio e suporte técnico à implantação dos complexos reguladores municipais também fizesse parte do elenco dos indicadores consensuais nessa dimensão. Pereira² reforça esse aspecto quando destaca como função do gestor estadual o fortalecimento da capacidade institucional de municípios e órgãos de gestão regiona-lizada, por meio de apoio técnico, político e financeiro. Contudo, a independência no julgamento dos critérios e dos indicadores pelos especialistas, a partir da metodologia adotada, permite uma infinidade de arranjos possíveis para o consenso.

A incorporação ou desenvolvimento de tecnologias de gestão mostrou-se consensual para os três indicadores de avaliação propostos. Entendeu-se que novas tecnologias contribuem para aumentar a eficiência e a eficácia das políticas e dos processos gerencias, bem como aumentam o potencial de utilização das informações, de forma a ter disponível informações clínicas, epidemiológicas e administrativas que orientem as ações.

$\mathrm{Na}$ dimensão participação e controle social mostraram-se consensuais os indicadores de avaliação do gestor estadual que se referem ao aperfeiçoamento do exercício do controle social nos espaços regionais como a implantação de ouvidorias nos estados e municípios, a implementação de conselhos gestores de unidades de referência regional do sistema, a realização de plenárias regionais e o incentivo aos processos de formação de conselheiros.

Cabe destacar aqui que não foram consensuais os critérios referentes ao acompanhamento dos instrumentos de planejamento regional por parte dos Conselhos e à participação destes na contratualização dos prestadores de serviços. Tal entendimento partiu da compreensão de alguns especialistas de que o papel dos Conselhos de Saúde não deve ser técnico, mas voltado para aspectos mais gerais das políticas de saúde, definindo as diretrizes gerais de formulação dessas políticas. Assim, a delegação de tarefas que são essencialmente técnicas desqualifica a participação de conselheiros, fazendo com que estes se sintam, muitas vezes, incapazes de atuar.

\section{Nível de análise: Assistência}

Os critérios e indicadores de avaliação nesse nível de análise foram avaliados como importantes, obtendo amplo consenso entre os especialistas, talvez por abordar os aspectos mais técnicos e normatizados de todas as categorias de análise, embora a questão da qualidade em saúde possa ser abordada sob diferentes perspectivas.

$\mathrm{Na}$ dimensão qualidade da assistência, a relação dos indicadores de avaliação está apoiada na proposta de Donabedian, 27 que concebe a tríade estrutura-processo-resultado como modelo de avali- 
ação da qualidade. Nesta abordagem, a estrutura consiste dos inputs necessários aos processos de cuidados em saúde, ou seja, força de trabalho, instalações físicas, equipamentos e demais insumos adequados a padrões estabelecidos. $\mathrm{O}$ processo consiste na adequação das práticas em saúde, isto é, na sua conformidade frente aos conhecimentos técnico-científicos vigentes. $\mathrm{O}$ resultado consiste na aferição dos objetivos pretendidos com os cuidados em saúde. Neste estudo, o resultado é a única dimensão da tríade que não foi incluída, já que a matriz de avaliação enfatiza os indicadores de estrutura e de processo relacionados à regionalização.

A maioria dos indicadores obteve elevado grau de importância no julgamento dos especialistas, sendo especialmente relevante avaliar o papel do gestor estadual no que tange à adequação dos serviços ao nível de complexidade assistencial a que se propõem dentro do sistema de saúde. Para Dubeux et al.,28 tal aspecto deve considerar intervenções governamentais estruturadas na direção da suficiência de profissionais, equipamentos e materiais essenciais, bem como do enfrentamento das fragilidades persistentes dos vínculos e das oportunidades de qualificação profissional, que devem ser fortalecidas na direção da interiorização.

Tem-se claro, contudo, que para os especialistas, a identificação desses indicadores não esgota a discussão da qualidade da assistência, mas constitui uma tentativa de traduzir a necessidade de avaliar como o gestor estadual atua na busca da qualidade da assistência prestada, de forma a garantir a resolutividade da atenção nas regiões de saúde.

Em relação à dimensão educação em saúde, esta foi entendida enquanto um papel fundamental do gestor estadual no tocante à gestão regionalizada do SUS, devendo se avançar na implementação de uma política de educação permanente como estratégia para a formação e o desenvolvimento dos trabalhadores da saúde, considerando as especificidades regionais e as necessidades de formação para o SUS. 26

Para isso, a política de educação permanente em saúde deve considerar a capacidade já instalada de oferta institucional de ações formais de educação na saúde, cuja execução deve se dar por meio dos colegiados de gestão regional, com a participação das Comissões Permanentes de Integração EnsinoServiço (CIES), como instâncias intersetoriais e interinstitucionais permanentes que devem atuar na formulação, condução e desenvolvimento da política de educação permanente em saúde. 29

É importante destacar que o esforço empreendido na construção de um modelo de avaliação da implantação da regionalização da assistência no SUS foi pautado pela necessidade de maior compreensão do papel do gestor estadual, já que as diferentes dimensões que evidenciam os processos políticos e gerenciais implicados na sua operacionalização não estão facilmente explicitadas nas normas, mostrando uma insuficiência dos dispositivos legais para tornálas efetivas e praticáveis, o que vem a refletir no grau com que as ações são implantadas.

Além disso, a elaboração de modelos de avaliação e o uso de técnicas de construção de consenso por outros estudos $15,18,19$ aponta no sentido de uma tendência cada vez mais crescente no uso dessa ferramenta, enquanto prática de investigação científica.

\section{Conclusões}

O modelo de regionalização que se almeja para o SUS na visão de especialistas, aqui apresentado, cumpre a finalidade de explicitar os processos necessários e suficientes para produzirem isolada ou integradamente os efeitos que dele se espera. Tem-se claro que este modelo não é definitivo. Ele representa apenas certo grau de consenso provisório sobre o papel do gestor estadual na regionalização da assistência à saúde. Todavia, o consenso amplia a validade dos critérios e confere maior legitimidade aos processos avaliativos, contribuindo tanto para o desenvolvimento da investigação científica quanto do debate político em torno da reorganização setorial.

A matriz de avaliação consensuada poderá servir de referência para a realização de investigações avaliativas que busquem entender a implantação dos processos de regionalização em saúde, trabalhando com diversas estratégias metodológicas no sentido de aferir em que medida esse modelo está sendo alcançado. Poderá ainda permitir a comparação de resultados e, consequentemente, a construção de evidências científicas, quando da sua larga utilização. Aos gestores de saúde, pode servir como um importante instrumento, orientando sobre os processos-chave que devem ser implementados.

\section{Referências}

1. Mendes EV. Os grandes dilemas do SUS: tomo I e II. Salvador: Casa da Qualidade Editora; 2001.
2. Pereira AMM. Dilemas federativos e a regionalização na saúde: o papel do gestor estadual do SUS em Minas Gerais 
[dissertação]. Rio de Janeiro: Escola Nacional de Saúde Pública Sérgio Arouca; 2009.

3. Souza RR. A regionalização no contexto atual das políticas de saúde. Ciênc Saúde Coletiva. 2001; 2: 451-5.

4. Lucchese PTR. Descentralização do financiamento e gestão da assistência à saúde no Brasil: a implementação do Sistema Único de Saúde - retrospectiva 1990/1995. Planej Polít Públicas. 1996 [acesso em 5 fev 2007]. Disponível em: http://www.ipea.gov.br/pub/ppp/ppp14/patricia.pdf

5. Hartz ZMA, Contandriopoulos AP. Integralidade da atenção e integração de serviços de saúde: desafios para avaliar a implantação de um "sistema sem muros". Cad Saúde Pública. 2004; V (Supl. 2): 331-6.

6. Contandriopoulos AP, Champagne F, Denis JL, Pineault RA. Avaliação na área da saúde: conceitos e métodos. In: Hartz ZMA, org. Avaliação em saúde: dos modelos conceituais à prática na análise da implantação de programas. Rio de Janeiro: Fiocruz; 1997. p. 29-48.

7. Chen HT. The theory-driven perspective. In: Chen HT, org. Theory-driven evaluations. California: Sage Publications; 1990. p. 37-52.

8. Bezerra LCA, Cazarin G, Alves CKA. Modelagem de programas: da teoria à operacionalização. In: Samico I, Felisberto E, Figueiró AC, Frias PG, orgs. Avaliação em saúde: bases conceituais e operacionais. Rio de Janeiro: MedBook; 2010. p. 65-78.

9. Medina MG, Silva GAP, Aquino R, Hartz ZMA. Uso de modelos teóricos na avaliação em saúde: aspectos conceituais e operacionais. In: Hartz ZMA, Vieira-da-Silva LM, orgs. Avaliação em saúde: dos modelos teóricos à prática na avaliação de programas e sistemas de saúde. Salvador/Rio de Janeiro: EDUFBA/Fiocruz; 2005. p. 4163

10. Brasil. Conselho Nacional de Secretários de Saúde. A gestão da saúde nos estados: avaliação e fortalecimento das funções estaduais. Brasília, DF; 2007

11. Magalhães Jr HM. O desafio de construir e regular redes públicas com integralidade, em sistemas privado-dependentes: a experiência de Belo Horizonte [tese]. Campinas: Faculdade de Ciências Médicas da Universidade Estadual de Campinas; 2006.

12. Mattos RA. A integralidade na prática (ou sobre a prática da integralidade). Cad Saúde Pública. 2004; 20: 1411-6.

13. Ouverney AM. Regionalização do SUS: uma análise da estratégia de integração intermunicipal. Adm Diálogo. 2005; 7: 91-106.

14. Pestana M, Mendes EV. Pacto de Gestão: da municipalização autárquica à regionalização cooperativa. Belo Horizonte: Secretaria de Estado de Saúde de Minas Gerais; 2004

15. Sóter APM. O papel da esfera estadual na gestão descentralizada do Sistema Único de Saúde: o estudo de caso Pernambuco [dissertação]. Recife: Centro de Pesquisas Aggeu Magalhães da Fundação Oswaldo Cruz; 2009.

16. Souza LEPF, Silva LMV, Hartz ZMA. Conferência de consenso sobre a imagem-objetivo da descentralização da atenção à saúde no Brasil. In: Hartz ZMA, Vieira-da-Silva LM, orgs. Avaliação em saúde: dos modelos teóricos à prática na avaliação de programas e sistemas de saúde. Salvador/Rio de Janeiro: EDUFBA/Fiocruz; 2005. p. 65102.

17. Viacava F, Almeida C, Caetano R, Fausto M, Macinko J, Martins M, Noronha JC, Novaes HMD, Oliveira ES, Porto SM, Vieira-da-Silva LM, Szwarcwald CL. Uma metodologia de avaliação do desempenho do sistema de saúde brasileiro. Ciênc Saúde Coletiva. 2004; 3: 711-24.

18. Vieira-da-Silva LM, Hartz ZMA, Chaves SCL, Silva GAP, Paim JS. Análise da implantação da gestão descentralizada em saúde: estudo comparado de cinco casos na Bahia, Brasil. Cad Saúde Pública. 2007; 23: 355-70.

19. Alves CKA. Institucionalização da avaliação na atenção básica: análise do programa em uma gestão estadual [dissertação]. Recife: Centro de Pesquisas Aggeu Magalhães da Fundação Oswaldo Cruz; 2008.

20. Artmann E, Rivera FJU. Regionalização em saúde e mix público-privado [acesso em 9 dez 2009]. Disponível em: http://www.ans.gov.br/portal/upload/biblioteca/TT AS 05 _EArtmann_RegionalizaçãoEmSaude.pdf

21. Campos GWS. Um método para análise e co-gestão de coletivos - a construção do sujeito, a produção de valor de uso e a democracia em instituições: o método da roda. São Paulo: Hucitec; 2000.

22. Pelegrini MLM, Castro JD, Drachler ML. Equidade na alocação de recursos para a saúde: a experiência do Estado do Rio Grande do Sul, Brasil. Ciênc Saúde Coletiva. 2005; 2: $275-86$.

23. Cecílio LCO. Modelos tecno-assistenciais: da pirâmide ao círculo, uma possibilidade a ser explorada. Cad Saúde Pública. 1997; 3: 469-78.

24. Franco TB, Magalhães Jr. HM. Integralidade na assistência à saúde: a organização das linhas de cuidado. In: Merhy EE, Franco TB, orgs. O trabalho em saúde: olhando e experienciando o SUS no cotidiano. São Paulo: Hucitec; 2004. p. 125-33.

25. Brasil. Ministério da Saúde. Portaria $n^{\circ} 1.044$ de 01 de junho de 2004. Institui a Política Nacional para os hospitais de pequeno porte. Diário Oficial da União [DOU].2004. Brasília, DF. Disponível em: http://dtr2004.saude.gov.br/ hpp/documentos/Portaria_1044_2004.pdf

26. Brasil. Ministério da Saúde. Portaria no 699 de 30 de março de 2006. Regulamenta as diretrizes operacionais dos pactos pela vida e de gestão. Diário Oficial da União [DOU].2006. Brasília, DF. Disponível em: http://www.saude.sp.gov.br/ resources/gestor/destaques/port 699 regulamenta as diretrizes_operacionais_dos_pactos_pela_vida_e_de_gestao .pdf

27. Donabedian A. The definition of quality: a conceptual exploration. Explorations in quality assessment and monitoring: the definition of quality and approaches to its assessment. Michigan. 1988; 1: 3-31.

28. Dubeux LS, Carvalho EF, Reis YAC. Avaliação dos serviços de urgência e emergência da rede hospitalar de referência no Nordeste Brasileiro. Cad. Saúde Pública. 2010; 26: 1508-18

29. Brasil. Ministério da Saúde . Portaria $n^{\circ} 1.996$ de 20 de agosto de 2007. Dispõe sobre as diretrizes para a implemen- 
tação da política nacional de educação permanente em saúde. Diário Oficial da União [DOU]. 2007. Brasília, DF.

Disponível em: http://portal.saude.gov.br/portal/arquivos/

pdf/Portaria_1996-de_20_de_agosto-de-2007.pdf

Recebido em 22 de abril de 2010

Versão final apresntada em 20 de outubro de 2010

Aprovado em 25 de outubro de 2010 